PROCEEDINGS OF THE

AMERICAN MATHEMATICAL SOCIETY

Volume 140, Number 7, July 2012, Pages 2255-2265

S 0002-9939(2011)11090-6

Article electronically published on November 1,2011

\title{
IDEAL GAMES AND RAMSEY SETS
}

\author{
CARLOS DI PRISCO, JOSÉ G. MIJARES, AND CARLOS UZCÁTEGUI
}

(Communicated by Julia Knight)

\begin{abstract}
It is shown that Matet's characterization of the Ramsey property relative to a selective co-ideal $\mathcal{H}$, in terms of games of Kastanas, still holds if we consider semiselectivity instead of selectivity. Moreover, we prove that a coideal $\mathcal{H}$ is semiselective if and only if Matet's game-theoretic characterization of the $\mathcal{H}$-Ramsey property holds. This lifts Kastanas's characterization of the classical Ramsey property to its optimal setting, from the point of view of the local Ramsey theory, and gives a game-theoretic counterpart to a theorem of Farah, asserting that a co-ideal $\mathcal{H}$ is semiselective if and only if the family of $\mathcal{H}$-Ramsey subsets of $\mathbb{N}^{[\infty]}$ coincides with the family of those sets having the abstract $\mathcal{H}$-Baire property. Finally, we show that under suitable assumptions, for every semiselective co-ideal $\mathcal{H}$ all sets of real numbers are $\mathcal{H}$-Ramsey.
\end{abstract}

\section{INTRODUCTION}

Let $\mathbb{N}$ be the set of nonnegative integers. Given an infinite set $A \subseteq \mathbb{N}$, the symbol $A^{[\infty]}$ (resp. $A^{[<\infty]}$ ) represents the collection of the infinite (resp. finite) subsets of $A$. Let $A^{[n]}$ denote the set of all the subsets of $A$ with $n$ elements. If $\left.a \in \mathbb{N}^{[}<\infty\right]$ is an initial segment of $A \in \mathbb{N}^{[\infty]}$, then we write $a \sqsubset A$. Also, let $A / a:=\{n \in A: \max (a)<n\}$ and write $A / n$ to mean $A /\{n\}$. For $a \in \mathbb{N}^{[<\infty]}$ and $A \in \mathbb{N}^{[\infty]}$ let

$$
[a, A]:=\left\{B \in \mathbb{N}^{[\infty]}: a \sqsubset B \subseteq A\right\} .
$$

The family $\left\{[a, A]:(a, A) \in \mathbb{N}^{[<\infty]} \times \mathbb{N}^{[\infty]}\right\}$ is a basis for Ellentuck's topology, also known as exponential topology. In [2, Ellentuck gave a characterization of the Ramsey property in terms of the Baire property relative to this topology (see Theorem 2.1 below).

Let $(P, \leq)$ be a poset. A subset $D \subseteq P$ is dense in $P$ if for every $p \in P$, there is $q \in D$ with $q \leq p$. The subset $D \subseteq P$ is open if $p \in D$ and $q \leq p$ imply $q \in D$. We say $P$ is $\sigma$-distributive if the intersection of countably many dense open subsets of $P$ is dense. $P$ is $\sigma$-closed if every decreasing sequence of elements of $P$ has a lower bound.

Definition 1.1. A family $\mathcal{H} \subset \wp(\mathbb{N})$ is a co-ideal if it satisfies the following:

(i) $A \subseteq B$ and $A \in \mathcal{H}$ implies $B \in \mathcal{H}$ and

(ii) $A \cup B \in \mathcal{H}$ implies $A \in \mathcal{H}$ or $B \in \mathcal{H}$.

Received by the editors September 19, 2010 and, in revised form, February 19, 2011

2010 Mathematics Subject Classification. Primary 05D10; Secondary 03 E02.

Key words and phrases. Semiselective co-ideal, Ramsey theory, Kastanas games, BanachMazur games.

(C)2011 American Mathematical Society Reverts to public domain 28 years from publication 
The complement $\mathcal{I}=\wp(\mathbb{N}) \backslash \mathcal{H}$ is the dual ideal of $\mathcal{H}$. We will suppose that coideals differ from $\wp(\mathbb{N})$. Also, we say that a nonempty family $\mathcal{F} \subseteq \mathcal{H}$ is $\mathcal{H}$-disjoint if for every $A, B \in \mathcal{F}, A \cap B \notin \mathcal{H}$. We say that $\mathcal{F}$ is a maximal $\mathcal{H}$-disjoint family if it is $\mathcal{H}$-disjoint and it is not properly contained in any other $\mathcal{H}$-disjoint family.

A subset $\mathcal{X}$ of $\mathbb{N}^{[\infty]}$ is Ramsey if for every $[a, A] \neq \emptyset$ with $A \in \mathbb{N}^{[\infty]}$ there exists $B \in[a, A]$ such that $[a, B] \subseteq \mathcal{X}$ or $[a, B] \cap \mathcal{X}=\emptyset$. Some authors have used the term "completely Ramsey" to express this property, reserving the term "Ramsey" for a weaker property. Galvin and Prikry [4] showed that all Borel subsets of $\mathbb{N}^{[\infty]}$ are Ramsey, and Silver [12] extended this to all analytic sets. Mathias in [10] showed that if the existence of an inaccessible cardinal is consistent with $Z F C$, then it is consistent, with $Z F+D C$, that every subset of $\mathbb{N}^{[\infty]}$ is Ramsey. Mathias introduced the concept of a selective co-ideal (or a happy family), which has turned out to be of wide interest. Ellentuck [2] characterized the Ramsey sets as those having the Baire property with respect to the exponential topology of $\mathbb{N}^{[\infty]}$.

A game-theoretic characterization of the Ramsey property was given by Kastanas in [6], using games in the style of Banach-Mazur with respect to Ellentuck's topology.

In this work we will deal with a game-theoretic characterization of the following property:

Definition 1.2. Let $\mathcal{H} \subset \mathbb{N}^{[\infty]}$ be a co-ideal. $\mathcal{X} \subseteq \mathbb{N}^{[\infty]}$ is $\mathcal{H}$-Ramsey if for every $[a, A] \neq \emptyset$ with $A \in \mathcal{H}$ there exists $B \in[a, A] \cap \mathcal{H}$ such that $[a, B] \subseteq \mathcal{X}$ or $[a, B] \cap \mathcal{X}=\emptyset$. We say $\mathcal{X}$ is $\mathcal{H}$-Ramsey null if for every $[a, A] \neq \emptyset$ with $A \in \mathcal{H}$ there exists $B \in[a, A] \cap \mathcal{H}$ such that $[a, B] \cap \mathcal{X}=\emptyset$.

Mathias considered sets that are $\mathcal{H}$-Ramsey with respect to a selective co-ideal $\mathcal{H}$ and generalized Silver's result to this context. Matet 9 used games to characterize sets which are Ramsey with respect to a selective co-ideal $\mathcal{H}$. These games coincide with the games of Kastanas if $\mathcal{H}$ is $\mathbb{N}^{[\infty]}$ and with the games of Louveau $[8$ if $\mathcal{H}$ is a Ramsey ultrafilter.

Given a co-ideal $\mathcal{H} \subset \mathbb{N}^{[\infty]}$, the collection $\left\{[a, A]:(a, A) \in \mathbb{N}^{[<\infty]} \times \mathcal{H}\right\}$ is not, in general, a basis for a topology on $\mathbb{N}^{[\infty]}$, but the following abstract version of the Baire property and related concepts will be useful.

Definition 1.3. Let $\mathcal{H} \subset \mathbb{N}^{[\infty]}$ be a co-ideal. $\mathcal{X} \subseteq \mathbb{N}^{[\infty]}$ has the abstract $\mathcal{H}$-Baire property if for every $[a, A] \neq \emptyset$ with $A \in \mathcal{H}$ there exists $[b, B] \subseteq[a, A]$ with $B \in \mathcal{H}$ such that $[b, B] \subseteq \mathcal{X}$ or $[b, B] \cap \mathcal{X}=\emptyset$. We say $\mathcal{X}$ is $\mathcal{H}$-nowhere dense if for every $[a, A] \neq \emptyset$ with $A \in \mathcal{H}$ there exists $[b, B] \subseteq[a, A]$ with $B \in \mathcal{H}$ such that $[b, B] \cap \mathcal{X}=\emptyset$. Also, $\mathcal{X}$ is $\mathcal{H}$-meager if it is the union of countably many $\mathcal{H}$-nowhere dense sets.

Given a decreasing sequence $A_{0} \supseteq A_{1} \supseteq A_{2} \supseteq \cdots$ of infinite subsets of $\mathbb{N}$, a set $B$ is a diagonalization of the sequence (or $B$ diagonalizes the sequence) if and only if $B / n \subseteq A_{n}$ for each $n \in B$. A co-ideal $\mathcal{H}$ is selective if and only if every decreasing sequence in $\mathcal{H}$ has a diagonalization in $\mathcal{H}$.

A co-ideal $\mathcal{H}$ has the $Q^{+}$-property if for every $A \in \mathcal{H}$ and every partition $\left(F_{n}\right)_{n}$ of $A$ into finite sets, there is $S \in \mathcal{H}$ such that $S \subseteq A$ and $\left|S \cap F_{n}\right| \leq 1$ for every $n \in \mathbb{N}$.

Proposition $1.4([10])$. A co-ideal $\mathcal{H}$ is selective if and only if the poset $\left(\mathcal{H}, \subseteq^{*}\right)$ is $\sigma$-closed and $\mathcal{H}$ has the $Q^{+}$-property. 
Given a co-ideal $\mathcal{H}$, recall that a set $\mathcal{D} \subseteq \mathcal{H}$ is dense open in the ordering $(\mathcal{H}, \subseteq)$ if (a) for every $A \in \mathcal{H}$ there exists $B \in \mathcal{D}$ such that $B \subseteq A$ and (b) for every $A, B \in \mathcal{H}$, if $B \subseteq A$ and $A \in \mathcal{D}$, then $B \in \mathcal{D}$. Please notice that we will also consider the ordering $\left(\mathcal{H}, \subseteq^{*}\right)$, where $A \subseteq \subseteq^{*} B$ if and only if $A \backslash B$ is a finite set, but any reference to "dense open" in this paper will be only with respect to the ordering $(\mathcal{H}, \subseteq)$.

Given a sequence $\left\{D_{n}\right\}_{n \in \mathbb{N}}$ of dense open sets in $(\mathcal{H}, \subseteq)$, a set $B$ is a diagonalization of $\left\{D_{n}\right\}_{n \in \mathbb{N}}$ if and only if $B / n \in D_{n}$ for every $n \in B$. A co-ideal $\mathcal{H}$ is semiselective if for every sequence $\left\{D_{n}\right\}_{n \in \mathbb{N}}$ of dense open subsets of $\mathcal{H}$, the family of its diagonalizations is dense in $(\mathcal{H}, \subseteq)$.

Proposition $1.5([3])$. A co-ideal $\mathcal{H}$ is semiselective if and only if the poset $\left(\mathcal{H}, \subseteq^{*}\right)$ is $\sigma$-distributive and $\mathcal{H}$ has the $Q^{+}$-property.

Since $\sigma$-closedness implies $\sigma$-distributivity, then semiselectivity follows from selectivity, but the converse does not hold (see [3] for an example).

In Section 2 we list results of Ellentuck, Mathias and Farah that characterize topologically the Ramsey property and the local Ramsey property. In Section 3 we define a family of games and present the main result, which states that a co-ideal $\mathcal{H}$ is semiselective if and only if the $\mathcal{H}$-Ramsey sets are exactly those for which the associated games are determined. This generalizes results of Kastanas [6] and Matet [9]. The proof is given in Section 4. In Section 5] we show that in Solovay's model, for every semiselective co-ideal $\mathcal{H}$ all sets of real numbers from $L(\mathbb{R})$ are $\mathcal{H}$-Ramsey.

\section{Topological Characterization of the RAMSEy PROPERTy}

The following are the main results concerning the characterization of the Ramsey property and the local Ramsey property in topological terms.

Theorem 2.1 (Ellentuck). Let $\mathcal{X} \subseteq \mathbb{N}^{[\infty]}$ be given .

(i) $\mathcal{X}$ is Ramsey if and only if $\mathcal{X}$ has the Baire property with respect to Ellentuck's topology.

(ii) $\mathcal{X}$ is Ramsey null if and only if $\mathcal{X}$ is meager with respect to Ellentuck's topology.

Theorem 2.2 (Mathias). Let $\mathcal{X} \subseteq \mathbb{N}^{[\infty]}$ and let a selective co-ideal $\mathcal{H}$ be given.

(i) $\mathcal{X}$ is $\mathcal{H}$-Ramsey if and only if $\mathcal{X}$ has the abstract $\mathcal{H}$-Baire property.

(ii) $\mathcal{X}$ is $\mathcal{H}$-Ramsey null if and only if $\mathcal{X}$ is $\mathcal{H}$-meager.

Theorem 2.3 (Farah, Todorcevic). Let $\mathcal{H}$ be a co-ideal. The following are equivalent:

(i) $\mathcal{H}$ is semiselective.

(ii) The $\mathcal{H}$-Ramsey subsets of $\mathbb{N}^{[\infty]}$ are exactly those sets having the abstract $\mathcal{H}$ Baire property, and the following three families of subsets of $\mathbb{N}^{[\infty]}$ coincide and are $\sigma$-ideals:

(a) $\mathcal{H}$-Ramsey null sets,

(b) $\mathcal{H}$-nowhere dense sets, and

(c) $\mathcal{H}$-meager sets. 
In the next section we state results by Kastanas [6] and Matet [9] (Theorems 3.1 and 3.2 below) which are the game-theoretic counterparts of Theorems 2.1 and 2.2 . respectively, and we also present our main result (Theorem 3.3 below), which is the game-theoretic counterpart of Theorem 2.3 .

\section{Characterizing the RAmsey property with games}

The following is a relativized version of a game due to Kastanas [6], employed to obtain a characterization of the family of completely Ramsey sets (i.e. $\mathcal{H}$-Ramsey for $\mathcal{H}=\mathbb{N}^{[\infty]}$ ). The same game was used by Matet in $[9$ to obtain the analog result when $\mathcal{H}$ is selective.

Let $\mathcal{H} \subseteq \mathbb{N}^{[\infty]}$ be a fixed co-ideal. For each $\mathcal{X} \subseteq \mathbb{N}^{[\infty]}, A \in \mathcal{H}$ and $a \in \mathbb{N}^{[<\infty]}$ we define a two-player game $G_{\mathcal{H}}(a, A, \mathcal{X})$ as follows: player I chooses an element $A_{0} \in \mathcal{H} \uparrow A$; II answers by playing $n_{0} \in A_{0}$ such that $a \subseteq n_{0}$, and $B_{0} \in \mathcal{H} \cap$ $\left(A_{0} / n_{0}\right)^{[\infty]}$; then I chooses $A_{1} \in \mathcal{H} \cap B_{0}^{[\infty]}$; II answers by playing $n_{1} \in A_{1}$ and $B_{1} \in \mathcal{H} \cap\left(A_{1} / n_{1}\right)^{[\infty]}$; and so on. Player I wins if and only if $a \cup\left\{n_{j}: j \in \mathbb{N}\right\} \in \mathcal{X}$ :

$$
\begin{aligned}
& \begin{array}{lllllll}
I & A_{0} & A_{1} & \cdots & A_{k} & \ldots
\end{array} \\
& \text { II } \quad n_{0}, B_{0} \quad n_{1}, B_{1} \quad \cdots \quad n_{k}, B_{k} \quad \cdots
\end{aligned}
$$

A strategy for a player is a rule that tells him (or her) what to play based on the previous moves. A strategy is a winning strategy for player I if player I wins the game whenever he (or she) follows the strategy, no matter what player II plays. Analogously, it can be defined what a winning strategy for player II is. The precise definitions of strategy for two-player games can be found in [7, 11.

Let $s=\left\{s_{0}, \ldots, s_{k}\right\}$ be a nonempty finite subset of $\mathbb{N}$, written in its increasing order, and let $\vec{B}=\left\{B_{0}, \ldots, B_{k}\right\}$ be a sequence of elements of $\mathcal{H}$. We say that the pair $(s, \vec{B})$ is a legal position for player II if $\left(s_{0}, B_{0}\right), \ldots,\left(s_{k}, B_{k}\right)$ is a sequence of possible consecutive moves of II in the game $G_{\mathcal{H}}(a, A, \mathcal{X})$, respecting the rules. In this case, if $\sigma$ is a winning strategy for player I in the game, we say that $\sigma(s, \vec{B})$ is a realizable move of player $\mathbf{I}$ according to $\sigma$. Notice that if $r \in B_{k} / s_{k}$ and $C \in \mathcal{H}\left\lceil B_{k} / s_{k}\right.$, then $\left(s_{0}, B_{0}\right), \ldots,\left(s_{k}, B_{k}\right),(r, C)$ is also a sequence of possible consecutive moves of II in the game. We will sometimes use the notation $(s, \vec{B}, r, C)$ and say that $(s, \vec{B}, r, C)$ is a legal position for player II and $\sigma(s, \vec{B}, r, C)$ is a realizable move of player I according to $\sigma$.

We say that the game $G_{\mathcal{H}}(a, A, \mathcal{X})$ is determined if one of the players has a winning strategy.

Theorem 3.1 (Kastanas). $\mathcal{X}$ is Ramsey if and only if for every $A \in \mathbb{N}^{[\infty]}$ and $a \in \mathbb{N}^{[<\infty]}$ the game $G_{\mathbb{N}[\infty]}(a, A, \mathcal{X})$ is determined.

Theorem 3.2 (Matet). Let $\mathcal{H}$ be a selective co-ideal. $\mathcal{X}$ is $\mathcal{H}$-Ramsey if and only if for every $A \in \mathcal{H}$ and $a \in \mathbb{N}^{[<\infty]}$ the game $G_{\mathcal{H}}(a, A, \mathcal{X})$ is determined.

Now we state our main result:

Theorem 3.3. Let $\mathcal{H}$ be a co-ideal. The following are equivalent:

(1) $\mathcal{H}$ is semiselective.

(2) $\forall \mathcal{X} \subseteq \mathbb{N}^{[\infty]}, \mathcal{X}$ is $\mathcal{H}$-Ramsey if and only if for every $A \in \mathcal{H}$ and $a \in \mathbb{N}^{[<\infty]}$ the game $G_{\mathcal{H}}(a, A, \mathcal{X})$ is determined. 
So Theorem 3.3 is a game-theoretic counterpart to Theorem 2.3 in the previous section, in the sense that it gives us a game-theoretic characterization of semiselectivity. Obviously, it also gives us a characterization of the $\mathcal{H}$-Ramsey property, for semiselective $\mathcal{H}$, which generalizes the main results of Kastanas in [6] and Matet in [9] (Theorems 3.1 and 3.2 above).

It is known that every analytic set is $\mathcal{H}$-Ramsey for $\mathcal{H}$ semiselective (see Theorem 2.2 in $\left[3\right.$ or Lemma 7.18 in [14]). Assuming $A D_{\mathbb{R}}$, i.e., the axiom of determinacy for games over the reals (see [7] or [11]), we obtain the following from Theorem 3.3 .

Corollary 3.4. Assume $A D_{\mathbb{R}}$. If $\mathcal{H}$ is a semiselective co-ideal, then every subset of $\mathbb{N}[\infty]$ is $\mathcal{H}$-Ramsey.

\section{Proof of the main Result}

Throughout the rest of this section, fix a semiselective co-ideal $\mathcal{H}$. Before proving Theorem 3.3 in Propositions 4.1 and 4.7 below we will deal with winning strategies of players in a game $G_{\mathcal{H}}(a, A, \mathcal{X})$.

Proposition 4.1. For every $\mathcal{X} \subseteq \mathbb{N}^{[\infty]}, A \in \mathcal{H}$ and $a \in \mathbb{N}^{[<\infty]}$, I has a winning strategy in $G_{\mathcal{H}}(a, A, \mathcal{X})$ if and only if there exists $E \in \mathcal{H}\lceil A$ such that $[a, E] \subseteq \mathcal{X}$.

Proof. Suppose $\sigma$ is a winning strategy for I. We will suppose that $a=\emptyset$ and $A=\mathbb{N}$ without loss of generality.

Let $A_{0}=\sigma(\emptyset)$ be the first move of I using $\sigma$. We will define a tree $T$ of finite subsets of $A_{0}$, and for each $s \in T$ we will also define a family $M_{s} \subseteq A_{0}^{[\infty]}$ and a family $N_{s} \subseteq\left(A_{0}^{[\infty]}\right)^{|s|}$, where $|s|$ is the length of $s$. Put $\{p\} \in T$ for each $p \in A_{0}$ and let

$$
M_{\{p\}} \subseteq\left\{\sigma(p, B): B \in \mathcal{H} \uparrow A_{0}\right\}
$$

be a maximal $\mathcal{H}$-disjoint family (see paragraph after Definition[1.1), and set

$$
N_{\{p\}}=\left\{\{B\}: \sigma(p, B) \in M_{\{p\}}\right\} .
$$

Suppose we have defined $T \cap A_{0}^{[n]}$ and we have chosen a maximal $\mathcal{H}$-disjoint family $M_{s}$ of realizable moves of player I of the form $\sigma(s, \vec{B})$ for every $s \in T \cap A_{0}^{[n]}$. Let

$$
N_{s}=\left\{\vec{B}: \sigma(s, \vec{B}) \in M_{s}\right\}
$$

Given $s \in T \cap A_{0}^{[n]}, \vec{B} \in N_{s}$ and $r \in \sigma(s, \vec{B}) / s$, we put $s \cup\{r\} \in T$. Then choose a maximal $\mathcal{H}$-disjoint family

$$
M_{s \cup\{r\}} \subseteq\left\{\sigma(s, \vec{B}, r, C): \vec{B} \in N_{s}, C \in \mathcal{H}\lceil\sigma(s, \vec{B}) / r\} .\right.
$$

Put

$$
N_{s \cup\{r\}}=\left\{(\vec{B}, C): \sigma(s, \vec{B}, r, C) \in M_{s \cup\{r\}}\right\} .
$$

Now, for every $s \in T$, let

$$
\begin{gathered}
\mathcal{U}_{s}=\left\{E \in \mathcal{H}:\left(\exists F \in M_{s}\right) E \subseteq F\right\} \text { and } \\
\mathcal{V}_{s}=\left\{E \in \mathcal{H}:\left(\forall F \in M_{s \backslash\{\max (s)\}}\right) \max (s) \in F \rightarrow F \cap E \notin \mathcal{H}\right\} .
\end{gathered}
$$

Claim 4.2. For every $s \in T, \mathcal{U}_{s} \cup \mathcal{V}_{s}$ is dense open in $\left(\mathcal{H}\left\lceil A_{0}, \subseteq\right)\right.$. 
Proof. Fix $s \in T$ and $A \in \mathcal{H}\left\lceil A_{0}\right.$. If $\left(\forall F \in M_{s \backslash\{\max (s)\}}\right) \max (s) \in F \rightarrow F \cap A \notin \mathcal{H}$ holds, then $A \in \mathcal{V}_{s}$. Otherwise, fix $F \in M_{s \backslash\{\max (s)\}}$ such that $\max (s) \in F$ and $F \cap A \in \mathcal{H}$. Let $\vec{B} \in N_{s \backslash\{\max (s)\}}$ be such that $\sigma(s \backslash\{\max (s)\}, \vec{B})=F$. Notice that since $\max (s) \in F$, then

$$
(s \backslash\{\max (s)\}, \vec{B}, \max (s), F \cap A / \max (s))
$$

is a legal position for player II. Then, using the maximality of $M_{s}$, choose $\hat{F} \in M_{s}$ such that

$$
E:=\sigma(s \backslash\{\max (s)\}, \vec{B}, \max (s), F \cap A / \max (s)) \cap \hat{F}
$$

is in $\mathcal{H}$. So $E \in \mathcal{U}_{s}$ and $E \subseteq A$. This completes the proof of Claim 4.2 .

Claim 4.3. There exists $E \in \mathcal{H}\left\lceil A_{0}\right.$ such that for every $s \in T$ with $s \subset E$, $E / s \in \mathcal{U}_{s}$.

Proof. For each $n \in \mathbb{N}$, let

$$
\begin{aligned}
& \mathcal{D}_{n}=\bigcap_{\max (s)=n} \mathcal{U}_{s} \cup \mathcal{V}_{s}, \\
& \mathcal{U}_{n}=\bigcap_{\max (s)=n} \mathcal{U}_{s}
\end{aligned}
$$

(if there is no $s \in T$ with $\max (s)=n$, then we put $\mathcal{D}_{n}=\mathcal{U}_{n}=\mathcal{H}\left\lceil A_{0}\right.$ ). By Claim 4.2, every $\mathcal{D}_{n}$ is dense open in $\left(\mathcal{H} \uparrow A_{0}, \subseteq\right)$. Using semiselectivity, choose a diagonalization $\hat{E} \in \mathcal{H} \uparrow A_{0}$ of the sequence $\left(\mathcal{D}_{n}\right)_{n}$. Let

$$
E_{0}:=\left\{n \in \hat{E}: \hat{E} / n \in \mathcal{U}_{n}\right\} \text { and } E_{1}:=\hat{E} \backslash E_{0} .
$$

Let us prove that $E_{1} \notin \mathcal{H}$. Suppose $E_{1} \in \mathcal{H}$. By the definitions, $\left(\forall n \in E_{1}\right) \hat{E} / n \notin$ $\mathcal{U}_{n}$. Let $n_{0}=\min \left(E_{1}\right)$ and fix $s_{0} \subset \hat{E}$ such that $\max \left(s_{0}\right)=n_{0}$, satisfying, in particular, the following:

$$
\left(\forall F \in M_{s_{0} \backslash\left\{n_{0}\right\}}\right) n_{0} \in F \rightarrow F \cap E_{1} / n_{0} \notin \mathcal{H} .
$$

Notice that $\left|s_{0}\right|>1$, by the construction of the $M_{s}$ 's.

Now, let $m=\max \left(s_{0} \backslash\left\{n_{0}\right\}\right)$. Then $m \in E_{0}$ and therefore $\hat{E} / m \in \mathcal{U}_{m} \subseteq$ $\mathcal{U}_{s_{0} \backslash\left\{n_{0}\right\}}$. So there exists $F \in M_{s_{0} \backslash\left\{n_{0}\right\}}$ such that $\hat{E} / m \subseteq F$. Since $m<n_{0}$, $n_{0} \in F$. But $F \cap E_{1} / n_{0}=E_{1} / n_{0} \in \mathcal{H}$, a contradiction.

Hence, $E_{1} \notin \mathcal{H}$ and therefore $E_{0} \in \mathcal{H}$. Then $E:=E_{0}$ is as required.

Claim 4.4. Let $E$ be as in Claim 4.3 and let $s \cup\{r\} \in T$ with $s \subset E$ and $r \in E / s$. If $E / s \subseteq \sigma(s, \vec{B})$ for some $\vec{B} \in N_{s}$, then there exists $C \in \mathcal{H}\lceil\sigma(s, \vec{B}) / r$ such that $E / r \subseteq \sigma(s, \vec{B}, r, C)$ and $(\vec{B}, C) \in N_{s \cup\{r\}}$.

Proof. Fix $s$ and $r$ as in the hypothesis. Suppose $E / s \subseteq \sigma(s, \vec{B})$ for some $\vec{B} \in N_{s}$. Since $E / r \in \mathcal{U}_{s \cup\{r\}}$, there exists $(\vec{D}, C) \in N_{s \cup\{r\}}$ such that $E / r \subseteq \sigma(s, \vec{D}, r, C)$. Notice that $E / r \subseteq \sigma(s, \vec{B}) \cap \sigma(s, \vec{D})$. Since $M_{s}$ is $\mathcal{H}$-disjoint, then $\sigma(s, \vec{D})$ is neccesarily equal to $\sigma(s, \vec{B})$ and therefore $\sigma(s, \vec{B}, r, C)=\sigma(s, \vec{D}, r, C)$. Hence $(\vec{B}, C) \in N_{s \cup\{r\}}$ and $E / r \subseteq \sigma(s, \vec{B}, r, C)$.

Claim 4.5. Let $E$ be as in Claim 4.3 , Then $[\emptyset, E] \subseteq \mathcal{X}$. 
Proof. Let $\left\{k_{i}\right\}_{i \geq 0} \subseteq E$ be given. Since $E / k_{0} \in \mathcal{U}_{\left\{k_{0}\right\}}$, there exists $B_{0} \in N_{\left\{k_{0}\right\}}$ such that $E / k_{0} \subseteq \sigma\left(k_{0}, B_{0}\right)$. Thus, by the choice of $E$ and applying Claim 4.4 iteratively, we prove that $\left\{k_{i}\right\}_{i \geq 0}$ is generated in a run of the game in which player I has used his winning strategy $\sigma$. Therefore $\left\{k_{i}\right\}_{i \geq 0} \in \mathcal{X}$.

The converse is trivial. This completes the proof of Proposition 4.1.

Now we turn to the case when player II has a winning strategy. The proof of the following is similar to the proof of Proposition 4.3 in [9]. First we show a result we will need in the sequel. It should be compared with Lemma 4.2 in 9 .

Lemma 4.6. Let $B \in \mathcal{H}, f: \mathcal{H} \uparrow B \rightarrow \mathbb{N}$, and $g: \mathcal{H} \uparrow B \rightarrow \mathcal{H} \uparrow B$ be given such that $f(A) \in A$ and $g(A) \subseteq A / f(A)$. Then there is $E_{f, g} \in \mathcal{H} \uparrow B$ with the property that for each $p \in E_{f, g}$ there exists $A \in \mathcal{H} \uparrow B$ such that $f(A)=p$ and $E_{f, g} / p \subseteq g(A)$.

Proof. For each $n \in\{f(A): A \in \mathcal{H}\lceil B\}$, let

$$
U_{n}=\{E \in \mathcal{H} \uparrow B:(\exists A \in \mathcal{H} \uparrow B)(f(A)=n \wedge E \subseteq g(A))\}
$$

and

$$
V_{n}=\{E \in \mathcal{H} \uparrow B:(\forall A \in \mathcal{H}\lceil B)(f(A)=n \rightarrow|g(A) \backslash E|=\infty)\} .
$$

The set $D_{n}=U_{n} \cup V_{n}$ is dense open in $\mathcal{H} \uparrow B$. Choose $E \in \mathcal{H} \uparrow B$ such that for each $n \in E, E / n \in D_{n}$. Let

$$
E_{0}=\left\{n \in E: E / n \in U_{n}\right\} \text { and } E_{1}=\left\{n \in E: E / n \in V_{n}\right\} .
$$

Now, suppose $E_{1} \in \mathcal{H}$. Then, for each $n \in E_{1}, E_{1} / n \in V_{n}$. Let $n_{1}=f\left(E_{1}\right)$. So $n_{1} \in E_{1}$ by the definition of $f$. But, by the definition of $g, g\left(E_{1}\right) \subseteq E_{1} / n_{1}$ and so $E_{1} / n_{1} \notin V_{n_{1}}$, a contradiction. Therefore, $E_{1} \notin \mathcal{H}$. Hence $E_{0} \in \mathcal{H}$, since $\mathcal{H}$ is a co-ideal. The set $E_{f, g}:=E_{0}$ is as required.

Proposition 4.7. For every $\mathcal{X} \subseteq \mathbb{N}^{[\infty]}, A \in \mathcal{H}$ and $a \in \mathbb{N}^{[<\infty]}$, II has a winning strategy in $G_{\mathcal{H}}(a, A, \mathcal{X})$ if and only if $\forall A^{\prime} \in \mathcal{H}\left\lceil A\right.$ there exists $E \in \mathcal{H}\left\lceil A^{\prime}\right.$ such that $[a, E] \cap \mathcal{X}=\emptyset$.

Proof. Let $\tau$ be a winning strategy for II in $G_{\mathcal{H}}(a, A, \mathcal{X})$ and let $A^{\prime} \in \mathcal{H}\lceil A$ be given. We are going to define a winning strategy $\sigma$ for $\mathrm{I}$, in $G_{\mathcal{H}}\left(a, A^{\prime}, \mathbb{N}[\infty] \backslash \mathcal{X}\right)$, in such a way that we will get the required result by means of Proposition 4.1. So, in a play of the game $G_{\mathcal{H}}\left(a, A^{\prime}, \mathbb{N}[\infty] \backslash \mathcal{X}\right)$, with II's successive moves being $\left(n_{j}, B_{j}\right)$, $j \in \mathbb{N}$, define $A_{j} \in \mathcal{H}$ and $E_{f_{j}, g_{j}}$ as in Lemma 4.6. for $f_{j}$ and $g_{j}$ such that

(1) for all $\hat{A} \in \mathcal{H}\left\lceil A^{\prime}\right.$,

$$
\left(f_{0}(\hat{A}), g_{0}(\hat{A})\right)=\tau(\hat{A}) ;
$$

(2) for all $\hat{A} \in \mathcal{H} \uparrow B_{j} \cap g_{j}\left(A_{j}\right)$,

$$
\left(f_{j+1}(\hat{A}), g_{j+1}(\hat{A})\right)=\tau\left(A_{0}, \cdots, A_{j}, \hat{A}\right) ;
$$

(3) $A_{0} \subseteq A^{\prime}$ and $A_{j+1} \subseteq B_{j} \cap g_{j}\left(A_{j}\right)$;

(4) $n_{j}=f_{j}\left(A_{j}\right)$ and $E_{f_{j}, g_{j}} / n_{j} \subseteq g_{j}\left(A_{j}\right)$. 
Now, let $\sigma(\emptyset)=E_{f_{0}, g_{0}}$ and $\sigma\left(\left(n_{0}, B_{0}\right), \cdots,\left(n_{j}, B_{j}\right)\right)=E_{f_{j+1}, g_{j+1}}$.

Conversely, let $A_{0}$ be the first move of I in the game. Then there exists $E \in \mathcal{H} \uparrow$ $A_{0}$ such that $[a, E] \cap \mathcal{X}=\emptyset$. We define a winning strategy for player II by letting him (or her) play (minE, $E \backslash\{\min E\}$ ) at the first turn and arbitrarily from there on.

We are ready now for the following:

Proof of Theorem 3.3. If $\mathcal{H}$ is semiselective, then part (2) of Theorem 3.3 follows from Propositions 4.1 and 4.7

Conversely, suppose part (2) holds and $\left(\mathcal{D}_{n}\right)_{n}$ is a sequence of dense open sets in $(\mathcal{H}, \subseteq)$. For every $a \in \mathbb{N}^{[<\infty]}$, let

$\mathcal{X}_{a}=\left\{B \in[a, \mathbb{N}]: B / a\right.$ diagonalizes some decreasing $\left(A_{n}\right)_{n}$ such that $\left.(\forall n) A_{n} \in \mathcal{D}_{n}\right\}$ and define

$$
\mathcal{X}=\bigcup_{a \in \mathbb{N}[<\infty]} \mathcal{X}_{a}
$$

Fix $A \in \mathcal{H}$ and $a \in \mathbb{N}^{[<\infty]}$ with $[a, A] \neq \emptyset$, and define a winning strategy $\sigma$ for player I in $G_{\mathcal{H}}(a, A, \mathcal{X})$, as follows: let $\sigma(\emptyset)$ be any element of $\mathcal{D}_{0}$ such that $\sigma(\emptyset) \subseteq A$. At stage $k$, if II's successive moves in the game are $\left(n_{j}, B_{j}\right), j \leq k$, let $\sigma\left(\left(n_{0}, B_{0}\right), \ldots,\left(n_{k}, B_{k}\right)\right)$ be any element of $\mathcal{D}_{k+1}$ such that $\sigma\left(\left(n_{0}, B_{0}\right), \ldots,\left(n_{k}, B_{k}\right)\right)$ $\subseteq B_{k}$. Notice that $a \cup\left\{n_{0}, n_{1}, n_{2}, \ldots\right\} \in \mathcal{X}_{a}$.

So the game $G_{\mathcal{H}}(a, A, \mathcal{X})$ is determined for every $A \in \mathcal{H}$ and $a \in \mathbb{N}^{[}[<\infty]$ with $[a, A] \neq \emptyset$. Then, by our assumptions, $\mathcal{X}$ is $\mathcal{H}$-Ramsey. So given $A \in \mathcal{H}$, there exists $B \in \mathcal{H}\left\lceil A\right.$ such that $B^{[\infty]} \subseteq \mathcal{X}$ or $B^{[\infty]} \cap \mathcal{X}=\emptyset$. The second alternative does not hold, so $\mathcal{X} \cap \mathcal{H}$ is dense in $(\mathcal{H}, \subseteq)$. Hence, $\mathcal{H}$ is semiselective.

\section{The Ramsey property in Solovay models}

Recall that the Mathias forcing notion $\mathbb{M}$ is the collection of all the sets of the form

$$
[a, A]:=\left\{B \in \mathbb{N}^{[\infty]}: a \sqsubset B \subseteq A\right\},
$$

ordered by $[a, A] \leq[b, B]$ if and only if $[a, A] \subseteq[b, B]$.

If $\mathcal{H}$ is a co-ideal, then $\mathbb{M}_{\mathcal{H}}$, the Mathias partial order with respect to $\mathcal{H}$, is the collection of all the $[a, A]$ as above but with $A \in \mathcal{H}$, ordered in the same way.

A co-ideal $\mathcal{H}$ has the Mathias property if it satisfies the following: if $x$ is $\mathbb{M}_{\mathcal{H}^{-}}$ generic over a model $M$, then every $y \in x^{[\infty]}$ is $\mathbb{M}_{\mathcal{H}}$-generic over $M$. Also, $\mathcal{H}$ has the Prikry property if for every $[a, A] \in \mathbb{M}_{\mathcal{H}}$ and every formula $\varphi$ of the forcing language of $\mathbb{M}_{\mathcal{H}}$, there is $B \in \mathcal{H} \uparrow A$ such that $[a, B]$ decides $\varphi$.

Theorem 5.1 ([3, Theorem 4.1]). For a co-ideal $\mathcal{H}$ the following are equivalent:

(1) $\mathcal{H}$ is semiselective.

(2) $\mathbb{M}_{\mathcal{H}}$ has the Prikry property.

(3) $\mathbb{M}_{\mathcal{H}}$ has the Mathias property.

Suppose $M$ is a model of $Z F C$ and there is an inaccessible cardinal $\lambda$ in $M$. The Levy partial order $\operatorname{Col}(\omega,<\lambda)$ produces a generic extension $M[G]$ of $M$ where $\lambda$ becomes $\aleph_{1}$. Solovay's model (see [13]) is obtained by taking the submodel of $M[G]$ formed by all the sets hereditarily definable in $M[G]$ from a sequence of ordinals (see [10] or [5]). 
In [10, Mathias shows that if $V=L, \lambda$ is a Mahlo cardinal and $V[G]$ is a generic extension obtained by forcing with $\operatorname{Col}(\omega,<\lambda)$, then every set of real numbers defined in the generic extension from a sequence of ordinals is $\mathcal{H}$-Ramsey for $\mathcal{H}$ a selective co-ideal. This result can be extended to semiselective co-ideals under a suitable large cardinal hypothesis.

Theorem 5.2. Suppose $\lambda$ is a weakly compact cardinal. Let $V[G]$ be a generic extension by $\operatorname{Col}(\omega,<\lambda)$. Then, if $\mathcal{H}$ is a semiselective co-ideal in $V[G]$, every set of real numbers in $L(\mathbb{R})$ of $V[G]$ is $\mathcal{H}$-Ramsey.

Proof. Let $\mathcal{H}$ be a semiselective co-ideal in $V[G]$. Let $\mathcal{A}$ be a set of reals in $L(\mathbb{R})^{V[G]}$; in particular, $\mathcal{A}$ is defined in $V[G]$ by a formula $\varphi$ from a sequence of ordinals. Let $[a, A]$ be a condition of the Mathias forcing $\mathbb{M}_{\mathcal{H}}$ with respect to the semiselective co-ideal $\mathcal{H}$. Finally, let $\dot{\mathcal{H}}$ be a name for $\mathcal{H}$. Notice that $\dot{\mathcal{H}} \subseteq V_{\lambda}$.

Since $V[G]$ satisfies that $\mathcal{H}$ is semiselective, the following statement holds in $V[G]$ : For every sequence $D=\left(D_{n}: n \in \omega\right)$ of open dense subsets of $\mathcal{H}$ and for every $x \in \mathcal{H}$ there is $y \in \mathcal{H}, y \subseteq x$, such that $y$ diagonalizes the sequence $D$.

Therefore, there is $p \in G$ such that, in $V$, the following statement holds:

$$
\begin{array}{r}
\forall \dot{D} \forall \tau\left(p \Vdash_{C o l(\omega,<\lambda)}(\dot{D} \text { is a name for a sequence of dense open subsets of } \dot{\mathcal{H}}\right. \\
\text { and } \tau \in \dot{\mathcal{H}}) \longrightarrow(\exists x(x \in \dot{\mathcal{H}}, x \subseteq \tau, x \text { diagonalizes } \dot{D}))) .
\end{array}
$$

Notice that every real in $V[G]$ has a name in $V_{\lambda}$, and names for subsets of $\mathcal{H}$ or countable sequences of subsets of $\mathcal{H}$ are contained in $V_{\lambda}$. Also, the forcing $\operatorname{Col}(\omega,<\lambda)$ is a subset of $V_{\lambda}$. Therefore the same statement is valid in the structure $\left(V_{\lambda}, \in, \dot{\mathcal{H}}, \operatorname{Col}(\omega,<\lambda)\right)$. This statement is $\Pi_{1}^{1}$ over this structure, and since $\lambda$ is $\Pi_{1}^{1}$ indescribable, there is $\kappa<\lambda$ such that in $\left(V_{\kappa}, \in, \dot{\mathcal{H}} \cap V_{\kappa}, \operatorname{Col}(\omega,<\lambda) \cap V_{\kappa}\right)$

$$
\begin{array}{r}
\forall \dot{D} \forall \tau\left(p \Vdash _ { C o l ( \omega , < \kappa ) } \left(\dot{D} \text { is a name for a sequence of dense open subsets of } \dot{\mathcal{H}} \cap V_{\kappa}\right.\right. \\
\text { and } \left.\left.\tau \in \dot{\mathcal{H}} \cap V_{\kappa}\right) \longrightarrow\left(\exists x\left(x \in \dot{\mathcal{H}} \cap V_{\kappa}, x \subseteq \tau, x \text { diagonalizes } \dot{D}\right)\right)\right) .
\end{array}
$$

We can get $\kappa$ inaccessible, since there is a $\Pi_{1}^{1}$ formula expressing that $\lambda$ is inaccessible. Also, $\kappa$ is such that $p$ and the names for the real parameters in the definition of $\mathcal{A}$ and for $A$ belong to $V_{\kappa}$.

If we let $G_{\kappa}=G \cap \operatorname{Col}(\omega,<\kappa)$, then $G_{\kappa} \subseteq \operatorname{Col}(\omega,<\kappa)$ and it is generic over $V$. Also, $p \in G_{\kappa}$. Here $\dot{\mathcal{H}} \cap V_{\kappa}$ is a $\operatorname{Col}(\omega,<\kappa)$-name in $V$ which is interpreted by $G_{\kappa}$ as $\mathcal{H} \cap V\left[G_{\kappa}\right]$; thus $\mathcal{H} \cap V\left[G_{\kappa}\right] \in V\left[G_{\kappa}\right]$. Moreover, since every subset (or sequence of subsets) of $\mathcal{H} \cap V\left[G_{\kappa}\right]$ which belongs to $V\left[G_{\kappa}\right]$ has a name contained in $V_{\kappa}$, we have that, in $V\left[G_{\kappa}\right], \mathcal{H} \cap V_{\kappa}$ is semiselective, and in consequence it has both the Prikry and the Mathias properties.

Now the proof can be finished as in [10]. Let $\dot{r}$ be the canonical name of an $\mathbb{M}_{\mathcal{H} \cap V\left[G_{\kappa}\right]}$-generic real and consider the formula $\varphi(\dot{r})$ in the forcing language of $V\left[G_{\kappa}\right]$. By the Prikry property of $\mathcal{H} \cap V\left[G_{\kappa}\right]$, there is $A^{\prime} \subseteq A, A^{\prime} \in \mathcal{H} \cap V\left[G_{\kappa}\right]$, such that $\left[a, A^{\prime}\right]$ decides $\varphi(\dot{r})$. Since $2^{2^{\omega}}$ computed in $V\left[G_{\kappa}\right]$ is countable in $V[G]$, there is (in $V[G]$ ) an $\mathbb{M}_{\mathcal{H} \cap V\left[G_{\kappa}\right]}$-generic real $x$ over $V\left[G_{\kappa}\right]$ such that $x \in\left[a, A^{\prime}\right]$. 
To see that there is such a generic real in $\mathcal{H}$ we argue as in 5.5 of [10] using the semiselectivity of $\mathcal{H}$ and the fact that $\mathcal{H} \cap V\left[G_{\kappa}\right]$ is countable in $V[G]$ to obtain an element of $\mathcal{H}$ which is generic. By the Mathias property of $\mathcal{H} \cap V\left[G_{\kappa}\right]$, every $y \in[a, x \backslash a]$ is also $\mathbb{M}_{\mathcal{H} \cap V\left[G_{\kappa}\right]}$-generic over $V\left[G_{\kappa}\right]$, and also $y \in\left[a, A^{\prime}\right]$. Thus $\varphi(x)$ if and only if $\left[a, A^{\prime}\right] \Vdash \varphi(\dot{r})$, if and only if $\varphi(y)$. Therefore, $[a, x \backslash a]$ is contained in $\mathcal{A}$ or is disjoint from $\mathcal{A}$.

As in [10, we obtain the following.

Corollary 5.3. If $Z F C$ is consistent with the existence of a weakly compact cardinal, then so is the statement that for every semiselective co-ideal $\mathcal{H}$ all sets of real numbers from $L(\mathbb{R})$ are $\mathcal{H}$-Ramsey.

Eisworth (1]) showed that the hypothesis of the existence of a Mahlo cardinal in Mathias's result cannot be weakened.

Question. Can the weakly compact cardinal hypothesis in the statement of Theorem 5.2 be weakened? Would a Mahlo cardinal suffice?

\section{ACKNOWLEDGMENTS}

The authors thank A. Blass, J. Bagaria and the referee for helping to correct some deficiencies in previous versions of the article.

\section{REFERENCES}

[1] Eisworth, T., Selective ultrafilters and $\omega \rightarrow(\omega)^{\omega}$, Proc. Amer. Math. Soc., 127 (1999) 30673071. MR.1600136 (2000a:03083)

[2] Ellentuck, E., A new proof that analytic sets are Ramsey, J. Symbolic Logic, 39 (1974), 163-165. MR0349393 (50:1887)

[3] Farah, I., Semiselective co-ideals. Mathematika, 45 (1997), 79-103. MR1644345|(2000b:03165)

[4] Galvin, F., and K. Prikry, Borel sets and Ramsey's theorem, J. Symbolic Logic, 38 (1973), 193-198. MR0337630(49:2399)

[5] Jech, T., Set Theory. Springer Verlag, 2003. MR1940513 (2004g:03071)

[6] Kastanas, I., On the Ramsey property for sets of reals. J. Symbolic Logic, 48 (1983), 10351045. MR727792 (85j:03080)

[7] Kechris, A.S., Classical Descriptive Set Theory. Springer-Verlag. Grad. Texts in Math. 156, 1995. MR1321597 (96e:03057)

[8] Louveau, A., Une méthode topologique pour l'étude de la propriété de Ramsey. Israel J. Math., 23 (1976), 97-116. MR0411971(54:100)

[9] Matet, P., Happy Families and Completely Ramsey Sets. Springer-Verlag, Archive for Mathematical Logic, 32 (1993), 151-171. MR1201647 (94i:03095)

[10] Mathias, A.R.D., Happy families. Annals of Mathematical Logic, 12 (1977), 59-111. MR0491197 (58:10462)

[11] Moschovakis, Y.N., Descriptive Set Theory. North-Holland, Amsterdam, 1980. MR.561709 (82e:03002)

[12] Silver, J., Every analytic set is Ramsey, J. Symbolic Logic, 35 (1970) 60-64. MR0332480 (48:10807)

[13] Solovay, R. M., A model of set-theory in which every set of reals is Lebesgue measurable. Annals of Mathematics (2), 92 (1970), 1-56. MR0265151(42:64)

[14] Todorcevic, S., Introduction to Ramsey Spaces, Princeton University Press, Princeton, New Jersey, 2010. MR2603812 
Instituto Venezolano de Investigaciones Científicas y Escuela de Matemática, Universidad Central de Venezuela, Caracas, Venezuela

E-mail address: cdiprisc@ivic.gob.ve

Instituto Venezolano de Investigaciones Científicas y Escuela de Matemática, Universidad Central de Venezuela, Caracas, Venezuela

E-mail address: jmijares@ivic.gob.ve, jose.mijares@ciens.ucv.ve

Current address: Departamento de Matematicas, Pontificia Universidad Javeriana, Bogota, Colombia

E-mail address: jmijares@javeriana.edu.co

Departamento de Matemáticas, Facultad de Ciencias, Universidad de los Andes, MÉrida, Venezuela

E-mail address: uzca@ula.ve 